\title{
Relativistic effect on low-energy nucleon-deuteron scattering
}

\author{
Sadhan K. Adhikari and Lauro Tomio \\ Instituto de Física Teórica, Universidade Estadual Paulista \\ 01405-000 São Paulo, São Paulo, Brasil
}

\begin{abstract}
The relativistic effect on differential cross sections, nucleon-to-nucleon and nucleonto-deuteron polarization transfer coefficients, and the spin correlation function, of nucleon-deuteron elastic scattering is investigated employing several three-dimensional relativistic three-body equations and several nucleon-nucleon potentials. The polarization transfer coefficients are found to be sensitive to the details of the nucleon-nucleon potentials and the relativistic dynamics employed, and prefer trinucleon models with the correct triton binding energy.
\end{abstract}

(To appear in Physical Review C)

\section{INTRODUCTION}

Recently, there have been many benchmark nonrelativistic calculations of the three-nucleon system involving

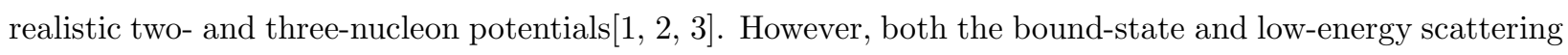
calculations of this system involve large momentum components of the wave function or the $t$ matrix elements, and this demands for a relativistic treatment of the problem. Relativistic dynamical calculations in the threenucleon problem have been mainly restricted to the study of the three-nucleon bound state [4, 5, 6, 6, 8, 9] with few exceptions where relativistic effect on the nucleon-deuteron (nd) scattering length has been studied [9, 10]. Both the four-dimensional Bethe-Salpeter-Faddeev equation四 5 in some approximate form and several types of three-dimensional reductions of this equation have been employed in numerical calculations [4, 5, 6, ,, , 9, 10]. 
We study the effect of relativistic dynamics on nd elastic scattering by employing several nucleon-nucleon potential models and four types of three-dimensional relativistic scattering equations suggested recently [11]. These relativistic equations obey conditions of relativistic covariance and two- and three-particle unitarity. At present time, one of the practical and feasible ways of performing a relativistic three-nucleon calculation is through these three-dimensional equations. At this point it should be noted that the solution of the approximate BS equation in ladder form, as has been performed recently [4, 5], is not necessarily a superior way of dealing with the relativistic effect[4, 12], partly because of the problems in interpreting the fourdimensional potential in the BS equation, and partly because of the difficulty in extracting a meaningful single-particle limit from the two particle BS equation in the ladder form.

In our previous investigation[9] we studied the effect of relativistic dynamics on the trinucleon binding energy and the $S$-wave nd scattering length. Here we study the relativistic effect on differential cross sections, various nucleon-to-nucleon and nucleon-to-deuteron polarization transfer coefficients, and the spin correlation function, $C_{x x}$ of nd elastic scattering. The present study indicates that these polarization transfer coefficients are very sensitive to the details of the nucleon-nucleon potentials and the dynamics employed in three-nucleon calculation, and favor models with correct triton binding energy.

The sensitivity of the nucleon-to-nucleon polarization transfer coefficients of nd elastic scattering, specially $K_{y}^{y^{\prime}}$, at low energies $(<10 \mathrm{MeV})$ to the off-shell behavior of the nucleon-nucleon potential has been known for quite some time13. Recently, in relation to a study of the three-nucleon problem with realistic mesontheoretic potentials it has been observed[1] 14] that these polarization transfer coefficients at $22.7 \mathrm{MeV}$ are very sensitive to the tensor part of the nucleon-nucleon potential. It was found that the theoretical calculation employing the Bonn A meson-theoretic potential reproduced the results of nucleon-to-nucleon polarization transfer coefficient $K_{y}^{y^{\prime}}$ better than the calculation based on the meson-theoretic Paris potential. As the tensor parts of these two potentials are very different, it was concluded [1, 14] that this finding supports the weak tensor force of the Bonn A potential as being more realistic than the stronger tensor force of the Paris potential.

A previous study [15] demonstrated that three-nucleon models based on central nucleon-nucleon potentials can reproduce nucleon-to-nucleon polarization transfer coefficient $K_{y}^{y^{\prime}}$ quite well, provided that these models also reproduce the correct triton binding energy. In view of this it is unlikely that the nucleon-to-nucleon polarization transfer coefficient $K_{y}^{y^{\prime}}$ should carry much new information about tensor nucleon-nucleon potentials which is not implicit in the results of the triton binding energy. The Bonn A tensor potential reproduces for triton binding energy $8.38 \mathrm{MeV}$, whereas the Paris potential yields 7.47 MeV. As the Bonn A potential reproduces the experimental triton binding energy $(8.48 \mathrm{MeV})$ better than the Paris potential, it also reproduces the above $K_{y}^{y^{\prime}}$ better than the Paris potential. This casts doubt on the conclusion about the superiority of the Bonn A tensor potential over that of the Paris potential[1] 14. In our previous study 15. only nonrelativistic three-nucleon models were used. In this study we would like to see if the inclusion of 
relativistic dynamics changes the general conclusions of Ref. [15.

Though the magnitudes of relativistic corrections to the triton binding energy, $B_{t}$, and the $S$-wave spindoublet nd scattering length, $a_{n d}$, as emphasized in previous studies, are interesting, it is most relevant to see if meaningful physics could be extracted from the relativistic treatment of the three-nucleon system. The nonrelativistic potential model calculations of these two observables employing meson-theoretic nucleonnucleon potentials 16] did not allow us to extract meaningful informations about the two- and three-nucleon interactions because of the correlated behavior of the observables directly sensitive to these interactions [3]. The question to ask at this stage is whether the relativistic treatment of the three-nucleon problem is expected to change the scenario.

We present the present model in Sec. II. In Sec. III we present numerical results. Finally, in Sec. IV a brief summary of the conclusions are given.

\section{Nucleon-deuteron models}

The models that we shall use have been developed recently [9, 11. Here we present a brief summary of these models.

The relativistic two-nucleon dynamics for a central $S$ wave potential is taken to be governed by the following partial-wave Blankenbecler-Sugar (BIS) equation 19

$$
t\left(q^{\prime}, q, k^{2}\right)=V\left(q^{\prime}, q\right)+4 \pi \int_{0}^{\infty} p^{2} d p \frac{m}{\omega_{p}} V\left(q^{\prime}, p\right) \frac{1}{k^{2}-p^{2}+i 0} t\left(p, q, k^{2}\right),
$$

where $\omega_{p}=\left(m^{2}+p^{2}\right)^{1 / 2}$. The nonrelativistic two-nucleon dynamics is taken to be governed by the LippmannSchwinger (LS) equation.

We take the relativistic nucleon-nucleon potential in the following form

$$
\left[V_{n}\left(q^{\prime}, q\right)\right]_{r e l}=-\lambda_{n}\left[v_{n}\left(q^{\prime}\right)\right]_{r e l}\left[v_{n}(q)\right]_{r e l},
$$

where $n=0$ (1) represents the spin triplet (singlet) state, and the subscript rel ( $n r)$ denotes relativistic (nonrelativistic).

The relativistic $t$ matrix in this case at the square of the center of mass (c.m.) energy $s=4\left(m^{2}+k^{2}\right)$ is given by

$$
\left[t_{n}\left(q^{\prime}, q, k^{2}\right)\right]_{r e l}=\left[v_{n}\left(q^{\prime}\right)\right]_{r e l}\left[\tau_{n}^{-1}\left(k^{2}\right)\right]_{r e l}\left[v_{n}(q)\right]_{r e l},
$$

where

$$
\left[\tau_{n}\left(k^{2}\right)\right]_{r e l}=-\frac{1}{\lambda_{n}}-4 \pi \int_{0}^{\infty} q^{2} d q\left(\frac{m}{\omega_{q}}\right) \frac{\left[v_{n}(q)\right]_{r e l}^{2}}{k^{2}-q^{2}+i 0} .
$$

We generate a nonrelativistic two-nucleon $t$ matrix, phase-equivalent to its relativistic version by the following transformation for the form-factors

$$
\left[v_{n}(q)\right]_{n r}=\left(\sqrt{m / \omega_{q}}\right)\left[v_{n}(q)\right]_{r e l},
$$


so that

$$
\left[t_{n}\left(q^{\prime}, q, s\right)\right]_{n r}=\left[v_{n}\left(q^{\prime}\right)\right]_{n r}\left[\tau_{n}^{-1}\left(k^{2}\right)\right]_{r e l}\left[v_{n}(q)\right]_{n r},
$$

The functional form of $[\tau]_{r e l}$ of Eq. (6) is exactly identical to its relativistic counterpart (4). This procedure generates phase-equivalent two-nucleon potentials to be used in nonrelativistic and relativistic three-nucleon problem.

The three-nucleon problem is solved with the following one-nucleon-exchange three-nucleon Born term[9]

$$
B_{n, n^{\prime}}(\vec{p}, \vec{q})=v_{n}(\mathcal{P}) v_{n^{\prime}}(\mathcal{Q}) G(\vec{p}, \vec{q}) .
$$

In the nonrelativistic case the propagator $G(\vec{p}, \vec{q})$ is given by,

$$
G_{n r}(\vec{p}, \vec{q}, E)=\left(p^{2}+q^{2}+p q x-m E-i 0\right)^{-1},
$$

with $\mathcal{P}^{2}=p^{2} / 4+q^{2}+p q x$, and $\mathcal{Q}^{2}=q^{2} / 4+p^{2}+p q x$, where $x$ is the cosine of the angle between $\vec{p}$ and $\vec{q}$.

In the relativistic case we use the following propagators in Eq. (7) [9, 11]

$$
\begin{gathered}
G_{\mathcal{A}}(\vec{p}, \vec{q}, s)=\frac{2\left(\omega_{p}+\omega_{q}+\omega_{p q}\right)}{\omega_{p q}\left[\left(\omega_{p}+\omega_{q}+\omega_{p q}\right)^{2}-s-i 0\right]} ; \\
G_{\mathcal{B}}(\vec{p}, \vec{q}, s)=\frac{2\left(\omega_{p}+\omega_{q}\right)}{\omega_{p q}\left[\left(\omega_{p}+\omega_{q}\right)^{2}-\left(\sqrt{s}-\omega_{p q}\right)^{2}-i 0\right]} ; \\
G_{\mathcal{C}}(\vec{p}, \vec{q}, s)=\frac{1}{\omega_{p q}\left[\omega_{p}+\omega_{q}+\omega_{p q}-\sqrt{s}-i 0\right]} ; \\
G_{\mathcal{D}}(\vec{p}, \vec{q}, s)=\frac{2\left(\omega_{q}+\omega_{p q}\right)}{\omega_{p q}\left[\left(\omega_{q}+\omega_{p q}\right)^{2}-\left(\sqrt{s}-\omega_{p}\right)^{2}-i 0\right]},
\end{gathered}
$$

with $\mathcal{P}^{2}=\left(\omega_{q}+\omega_{p q}\right)^{2} / 4-p^{2} / 4-m^{2}$, and $\mathcal{Q}^{2}=\left(\omega_{p}+\omega_{p q}\right)^{2} / 4-q^{2} / 4-m^{2}$. Here we use notations $\omega_{p}=\left(m^{2}+p^{2}\right)^{1 / 2}, \omega_{p q}=\left[m^{2}+(\vec{p}+\vec{q})^{2}\right]^{1 / 2}$, etc. The spin variables are treated nonrelativistically in all cases.

In Eqs. (9) - (12) the parameter $s$ is the square of the total c.m. energy of the three-particle system. All these propagators satisfy conditions of relativistic three-particle unitarity, governed by that part of the denominator in these propagators which corresponds to the pole for three-particle propagation in the intermediate state, e.g., at $\sqrt{s}=\omega_{p}+\omega_{q}+\omega_{p q}$. The condition of relativistic three-particle unitarity in these propagators is manifested in having the same residue at this pole. These equations also satisfy conditions of two-particle unitarity.

Equation (9) was advocated by Aaron, Amado, and Young 20] and obeys time-reversal symmetry, e.g. $G(\vec{p}, \vec{q}, s)=G(\vec{q}, \vec{p}, s)$. Equations (10) and (11) also have this virtue of Eq. (9). The propagator $G_{\mathcal{C}}$ was suggested long ago 21. It has been shown 22 that the propagator $G_{\mathcal{D}}$ follows from a suggestion by Ahmadzadeh and Tjon 21. However, the propagator $G_{\mathcal{D}}$ has never appeared in this form before. Previous numerical applications 团, 6, 6, 可 of this propagator used unnecessary approximations which violated conditions of unitarity and covariance 22. Physically, these propagators differ in the way the particle and 
antiparticle contributions appears in the kernel of the integral equation. In the propagator $\mathcal{C}$, for example, there is no antiparticle contribution.

\section{$3 \quad$ Numerical Results}

For two-nucleon separable potentials in spin-triplet and spin-singlet channels we take the following Yamaguchi and Tabakin form-factors[17, recently used by Rupp and Tjon[⿰]:

$$
\begin{gathered}
g_{Y}(q)=\frac{1}{q^{2}+\beta^{2}}, \\
g_{T}(q)=\frac{q^{2}+\nu^{2}}{q^{2}+\gamma^{2}} \times \frac{q_{c}^{2}-q^{2}}{\left(q^{2}+\beta^{2}\right)^{\kappa}}, \kappa=1.5,2 .
\end{gathered}
$$

The Yamaguchi potential will be referred to as $\mathrm{Y}$, and the Tabakin potential with $\kappa=1.5,2$ will be referred to as T-1.5 and T-2, respectively. The constants of these potentials are given in Ref. [9].

Tabakin-type nucleon-nucleon potentials yield nucleon-nucleon phase shifts in better agreement with experiment, which change sign at higher energies, compared to the Yamaguchi potential. If Tabakin-type potential is used in both ${ }^{3} S_{1}$ and ${ }^{1} S_{0}$ spin channels, it leads to a triton ground state of several hundred MeV's 18]. However, we shall use the Tabakin potential in one of the nucleon-nucleon spin channels and Yamaguchi in the other and this does not lead to a collapsed triton and leads to trinucleon observables in better agreement with experiment and realistic calculations. The numerical calculation is also simplified by an order of magnitude in this model. We perform this 'cheap' study with a view to conclude if a more realistic calculation is worth the price.

At positive energies the three-particle equations were solved by the technique of contour rotation [3]. In the relativistic case, as was already noted before 4 , 5n in the bound-state problem, more care was needed to obtain a converged result in the scattering calculation. Some 48 mesh points were needed for obtaining converged relativistic scattering results with a rotation angle of 5 degrees, whereas some 24 mesh points were enough to obtain the nonrelativistic scattering results to the same degree of precision.

We calculated the $S$ wave spin-doublet nd scattering length, $a_{n d}$, differential cross section, spin correlation function, nucleon-to-nucleon and nucleon-to-deuteron polarization transfer coefficients of nucleon-deuteron elastic scattering and the triton binding, $B_{t}$ in the nonrelativistic case as well as with each of the four versions of relativistic formulations $\mathcal{A}-\mathcal{D}$. Propagator $\mathcal{A}$ has been used before in numerical calculations of the three-nucleon problem $[$, 5,6 , 10]. To the best of our knowledge, propagators $\mathcal{B}, \mathcal{C}$, and $\mathcal{D}$ are new and have never been used before in the three-nucleon problem.

The results for triton binding energies and the $S$ wave doublet scattering lengths in the present models have recently appeared [9. All the relativistic propagators increase the triton binding energy, $B_{t}$, in relation to the nonrelativistic case, except propagator $\mathcal{C}$ which reduces the binding. This tendency, also observed 
in previous calculations $4,8,8$, has been justified recently by theoretical arguments [9]. The relativistic correction to $B_{t}$ varies from $-0.3 \mathrm{MeV}$ to $0.7 \mathrm{MeV}$ in different situations. We observed in numerical calculations the following general inequality

$$
\left(B_{t}\right)_{\mathcal{D}},\left(B_{t}\right)_{\mathcal{B}}>\left(B_{t}\right)_{\mathcal{A}}>\left(B_{t}\right)_{n r}>\left(B_{t}\right)_{\mathcal{C}}
$$

These results are summarized in Fig. 1 where we plot $B_{t}$ versus $a_{n d}$ for the present nonrelativistic and relativistic model calculations, as well as for many other nonrelativistic calculations taken from the literature [23, 24]. In nonrelativistic calculations a correlation was observed between $B_{t}$ and $a_{n d}$ [3, 25] as a result of on- and off- shell variations of two- and three-nucleon potentials. The relativistic calculations of Fig. 1 differ in employing different relativistic dynamics and nucleon-nucleon potentials. The trend of the relativistic calculations is identical to that of the nonrelativistic calculations. Hence, the effect of including relativistic dynamics in the three-nucleon problem can not be distinguished from the effect of varying the two- and three-nucleon potentials in nonrelativistic calculations.

Next we present results for some of the low-energy nd scattering observables. We chose to exhibit five models for nd elastic scattering covering a wide range of variation of triton binding energy. They are

Model A: nonrelativistic dynamics, triplet Y singlet Y, $B_{t}=10.65 \mathrm{MeV}$,

Model B: relativistic propagator $\mathcal{B}$, triplet T-2 singlet $\mathrm{Y}, B_{t}=8.34 \mathrm{MeV}$,

Model C: relativistic propagator $\mathcal{A}$, triplet T-2 singlet Y, $B_{t}=8.14 \mathrm{MeV}$,

Model D: relativistic propagator $\mathcal{C}$, triplet T-2 singlet $\mathrm{Y}, B_{t}=7.91 \mathrm{MeV}$,

Model E: nonrelativistic dynamics, triplet Y singlet T-2, $B_{t}=7.69 \mathrm{MeV}$.

Three of these models are relativistic and two nonrelativistic. Note that the models A, B, C, D, and E produce triton binding energy in monotonically decreasing order. We performed calculations for other combinations of nucleon-nucleon potentials and propagators which follow the general trend of results obtained with these five illustrative models.

In Fig. 2 we plot the elastic differential scattering cross section at nucleon laboratory energies, $E_{n}$, of 10, 22.7, and $100 \mathrm{MeV}$. At lower energies the relativistic effect on this observable is small and possibly could be ignored. At higher energies $(100 \mathrm{MeV})$ this effect is small at forward and backward angles, but could be reasonable near the minimum of the cross section. The nd elastic differential cross section is mainly dominated by the spin quartet state and no specific correlation of the cross section with the triton binding energy was observed. In the following we consider several scattering observables which are correlated with the triton binding energy.

It is most relevant to consider the nucleon-to-nucleon polarization transfer coefficient, $K_{y}^{y^{\prime}}$ of nd elastic scattering. In Refs. [13] it has been claimed that this observable at $E_{n}=10 \mathrm{MeV}$ is very sensitive to the offshell behavior of the nucleon-nucleon $S$ wave interaction. A calculation based on Yamaguchi nucleon-nucleon potential did not reproduce the experimental results for this observable whereas that based on a meson- 
theoretic nucleon-nucleon potential[16] could explain the experimental results. The correct off-shell behavior of the meson-theoretic potential was made responsible for this [13]. More recently, it has been claimed[1, 14] that this observable at $E_{n}=22.7 \mathrm{MeV}$ is very sensitive to the tensor force of the nucleon-nucleon interaction. A calculation using the Paris nucleon-nucleon potential could not reproduce the experimental results for this observable whereas that using the Bonn A potential could satisfactorily account for the experimental results. The 'correct' tensor force of the Bonn A potential has been made responsible for this [1, 14]. In Ref. [15] it was pointed out that $K_{y}^{y^{\prime}}$ is correlated with the triton binding energy $B_{t}$ (or the spin doublet nd scattering length $a_{n d}$ ) in a dynamical calculation and the experimental data for $K_{y}^{y^{\prime}}$ favors a three-nucleon model with the correct triton binding energy. In the study of Ref. [13] the meson-theoretic nucleon-nucleon potential yielded a $B_{t}$, and hence $K_{y}^{y^{\prime}}$, closer to experiment than the Yamaguchi nucleon-nucleon potential. In Ref. [14 14 the Bonn A potential yields a $B_{t}$, and hence $K_{y}^{y^{\prime}}$, closer to experiment than the Paris nucleon-nucleon potential. Let us see if the inclusion of the relativistic dynamics changes the above scenario.

In Fig. 3 we plot nucleon-to-nucleon polarization transfer coefficient $K_{y}^{y^{\prime}}$ of nd elastic scattering at $E_{n}$ $=10 \mathrm{MeV}$ for the nd models A, B, C, D, and E. The results at $22.7 \mathrm{MeV}$ for this observable are shown in Fig 4. We find that $K_{y}^{y^{\prime}}$ is sensitive to both the nucleon-nucleon potential models as well as to the dynamics employed. However, the minimum of $K_{y}^{y^{\prime}}$ at about $\theta_{c . m}$. $=110$ degrees is found to be correlated with the triton binding energy as in the nonrelativistic case 15. In view of the present result and that of Ref. [15] it is highly likely that the better agreement of the Bonn A potential calculations for $K_{y}^{y^{\prime}}$ with the experiment over that of the Paris potential calculation at $E_{n}=22.7 \mathrm{MeV}$ is due to more precise $B_{t}$ produced by the former potential. Also, as simple central potential models of nd scattering could reproduce the results for $K_{y}^{y^{\prime}}$ reasonably well, it seems unlikely that it will really provide new information about the tensor nucleonnucleon potential besides those already contained in the value of triton binding energy. At higher energies, for example, at $E_{n}=100 \mathrm{MeV}$, the sensitivity of $K_{y}^{y^{\prime}}$ to the dynamics is found to be highly reduced; all five models yield essentially the same result for $K_{y}^{y^{\prime}}$ independent of triton binding.

Next we exhibit in Fig. 5 the spin correlation function $C_{x x}$ at $10 \mathrm{MeV}$ for the five nd potential models. Again the results for $C_{x x}$ are sensitive to the nd models, and arround $\theta_{c . m}=110$ degrees the results follow the order of the triton binding energy. The experimental points in this case are taken from Ref. [26]. In this case the dispersion between the curves of the different models is mostly determined by the corresponding triton binding energy and independent of the nucleon-nucleon potential models and the three-particle propagators.

Recently, it has been observed[27 that the nucleon-to-deuteron polarization transfer coefficients of nd elastic scattering are also sensitive to nd models employed. In order to study this sensitivity we plot in Fig. 6 and 7 nucleon-to-deuteron coefficients $K_{y}^{y^{\prime}}$ and $K_{x}^{x^{\prime}}$ at $E_{n}=10 \mathrm{MeV}$ for nd elastic scattering, respectively. The results for different models are separated again according to the value of triton binding energy.

It has been pointed out in Ref. [15] that though a $S$ wave separable potential model give a good description of the polarization transfer coefficients of nd elastic scattering, tensor and higher partial waves 
of nucleon-nucleon potentials are needed for their accurate description. For an $S$ wave model one should have for the nucleon-to-nucleon polarization transfer coefficients

$$
K_{x}^{x^{\prime}}=-K_{y}^{y^{\prime}} \sin \theta_{l a b}=K_{y}^{y^{\prime}} \cos \theta_{l a b} .
$$

The differences

$$
\Delta K=-K_{x}^{x^{\prime}}-K_{y}^{y^{\prime}} \sin \theta_{l a b}
$$

and

$$
\Delta K^{\prime}=-K_{x}^{x^{\prime}}+K_{y}^{y^{\prime}} \cos \theta_{l a b},
$$

are good measures of the effect of noncentrality of the nucleon-nucleon potental. However, to quantize such effects from a study of $\Delta K$ and $\Delta K^{\prime}$ high precision experimental results are needed. As the functions $\Delta K$ and $\Delta K^{\prime}$ are supposed to carry informations about the tensor-force and higher partial waves of nucleon-nucleon interaction, experimentalists are encouraged to provide accurate data for these observables. In the absence of these interactions both $\Delta K$ and $\Delta K^{\prime}$ are identically zero. Numerical calculations employing meson-theoretic nucleon-nucleon potentials are essential for an accurate description of the polarization transfer coefficients. However, as the experimental polarization transfer coefficients have large error bars, both the differences $\Delta K$ and $\Delta K^{\prime}$ are small with even larger errors 115$]$. Consequently, an $S$-wave treatment of the problem for drawing general conclusions as has been done here is justified.

Though we exhibit here in the figures results for five specific models, we performed and studied the numerical calculations for several more models. The general trend found in the case of these five models was observed in all cases. It is well known that the present $S$ wave separable potential models do not give a good description of the nd scattering at low energies. In spite of this, we have drawn some general conclusions which should be valid in realistic situations. We summarize these conclusions in the next section.

\section{Conclusions}

In conclusion, this is the first systematic study of relativistic effect on the trinucleon bound state and scattering employing combinations of Yamaguchi and Tabakin type potentials for the singlet and triplet nucleon-nucleon channels and four types of relativistic three-particle scattering equations.

We find in the calculations employing relativistic dynamics that $B_{t}$ is correlated to $a_{n d}$ as in nonrelativistic model calculations with variation of nucleon-nucleon potentials on- and off-shell. Hence it will be difficult to separate the effect of such variation of potentials from that of introducing the relativistic dynamics. This confirms the existence of a shape-independent approximation to these observables even after inclusion of the relativistic effect[23]. 
We observe that the nucleon-to-nucleon and nucleon-to-deuteron polarization transfer coefficients of nd elastic scattering are very sensitive to the details of potential model and relativistic dynamics. This sensitivity should be highly reduced once differnent models yield the same triton binding energy. In view of this it is

highly improbable that the results for nucleon-to nucleon polarization transfer coefficient, $K_{y}^{y^{\prime}}$, favors the weak tensor force of the Bonn A potential as has been concluded by Clajus et al. recently 14]. From a study of the nd elastic differential cross section, mainly dominated by the spin quartet state, no specific correlation with the triton binding energy was observed. However, the low-energy spin-correlation function is found to be correlated with the triton binding energy.

Of course, there are other observables for the three-nucleon system, which should be directly sensitive to relativistic effect, such as the charge form factors. Because of the presence of the possible large effect of meson-exchange currents and of the non-nucleonic components in the nucleus, such observables are not easily tractable, and it has so far been difficult to draw model independent conclusion from studies of these observables [1, 2].

We are aware that there is an inherent flexibility in deciding on the relativistic dynamics, in treating the spin variables relativistically, and in deciding the correct form of two- and three-nucleon potentials. We are far from exhausting all possibilities. But the tendency of existing the shape-independent approximation is so strong that we do not believe the present conclusions to be so peculiar as to be of no general validity. Hence a relativistic framework may reduce the still existing discrepancy between theory and experiment, but this may not enhance our knowledge of the three-nucleon system.

We thank Dr. T. Frederico for stimulating discussions. LT thanks the European Centre for Theoretical Studies in Nuclear Physics and Related Areas (ECT) at Trento, Italy for its hospitality and local support. The work is supported in part by the Conselho Nacional de Desenvolvimento - Científico e Tecnológico (CNPq) of Brasil.

\section{References}

[1] W. Glöckle, H. Witala, and Th. Cornelius, Nucl. Phys. A508, 115c (1990).

[2] H. Witala, W. Glöckle, and H. Kamada, Phys. Rev. C 43, 1619 (1991); R. A. Brandenburg, G. S. Chulick, R. Machleidt, A. Picklesimer, and R. M. Thaler, ibid. 37, 1245 (1988); S. Ishikawa and T. Sasakawa, Few-Body Syst. 1, 145 (1986); J. L. Friar, B. F. Gibson, G. L. Payne, and S. A. Coon, ibid. 5, 13 (1988); J. Friar, Nucl. Phys. A463, 315c (1987); B. F. Gibson, ibid. A543, 1c (1992); T. Sasakawa, ibid. A463, 327c (1987); A. Kievsky, M. Viviani, and S. Rosati, ibid. A551, 241 (1993).

[3] S. K. Adhikari, and K. L. Kowalski, Dynamical Collision Theory and Its Applications, Academic Press, Boston, 1991. 
[4] G. Rupp and J. A. Tjon, Phys. Rev. C 45, 2133 (1992).

[5] G. Rupp and J. A. Tjon, Phys. Rev. C 37, 1729 (1988).

[6] H. Garcilazo, Phys. Rev. C 23, 559 (1981).

[7] F. Sammarruca, D. P. Xu, and R. Machleidt, Phys. Rev. C 46, 1636 (1992).

[8] J. Carlson, V. R. Pandharipande, and R. Schiavilla, Phys. Rev. C 47, 484 (1993); W. Glöckle, T. S. H. Lee, and F. Coester, ibid. 33, 709 (1986).

[9] S. K. Adhikari and L. Tomio, Ann. Phys. (N.Y.) xxx, xxx (1994).

[10] H. Garcilazo, L. Mathelitsch, and H. Zankel, Phys. Rev. C 32, 264 (1985).

[11] S. K. Adhikari, T. Frederico, and L. Tomio, Ann. Phys. (N.Y.) xxx, xxx (1994).

[12] F. Gross, Phys. Rev. C 26, 2203 (1982); Relativistic Quantum Mechanics and Field Theory, Wiley, New York, 1993.

[13] H. Zankel, W. Plessas, and J. Heidenbauer, Phys. Rev. C 28, 538 (1983); B. Loiseau, L. Mathelistch, W. Plessas, and K. Schwarz, ibid. 32, 2168 (1985); Y. Koike, W. Plessas, and H. Zankel, ibid. 32, 1796 (1985); H. Zankel and W. Plessas, Z. Phys. A 317, 45 (1984).

[14] M. Clajus et al., Phys. Lett. 245B, 333 (1990).

[15] T. Frederico, I. D. Goldman, and S. K. Adhikari, Phys. Rev. C 37, 949 (1988).

[16] R. Machleidt, K. Holinde, and Ch. Elster, Phys. Rep. 149, 1 (1987); M. Lacombe et al., Phys. Rev. C $21,861(1980)$.

[17] Y. Yamaguchi, Phys. Rev. 95, 1635 (1954); F. Tabakin, ibid. 174, 1208 (1968).

[18] A. Delfino, S. K. Adhikari, L. Tomio, and T. Frederico, Phys. Rev. C 46, 471, 1612 (1992).

[19] R. Blankenbecler and R. Sugar, Phys. Rev. 142, 1051 (1966).

[20] R. Aaron, R. D. Amado, and J. E. Young, Phys. Rev. 174, 2022 (1968).

[21] A. Ahmadzadeh and J. A. Tjon, Phys. Rev. 147, 1111 (1966).

[22] S. K. Adhikari, L. Tomio, and T. Frederico, Phys. Rev. C 48, 2105 (1993).

[23] L. Tomio, A. Delfino, and S. K. Adhikari, Phys. Rev. C 35, 441 (1987).

[24] S. K. Adhikari, Phys. Rev. C 30, 31 (1984).

[25] A. C. Phillips, Rep. Prog. Phys. 40, 905 (1977).

[26] J. Chauvin and J. Arvieux, Nucl. Phys. A247, 347 (1975). 
[27] T. Cornelius, W. Glöckle, J. Haidenbauer, Y. Koike, W. Plessas, and H. Witala, Phys. Rev. C 41, 2538 (1990).

\section{Figure Caption}

1. The $B_{t}$ versus $a_{n d}$ plot for various trinucleon models: the present relativistic models $(\diamond)$, the present nonrelativistic models $(+)$, and other nonrelativistic models taken from the literature $(\times)[24]$.

2. The nd elastic differential scattering cross section at different incident neucleon energies.

3. The nucleon-to-nucleon polarization transfer coefficient $K_{y}^{y^{\prime}}$ of nd elastic scattering for $E_{n}=10 \mathrm{MeV}$. The curves labelled A, B, and E correspond to models A, B, and E, respectively. The two curves between $\mathrm{B}$ and $\mathrm{E}$ correspond to models $\mathrm{C}$ and $\mathrm{D}$ in the order of decreasing triton binding energy. The dispersion between the curves is in the order of the variation of the triton binding energy.

4. Same as Fig. 3 for $E_{n}=22.7 \mathrm{MeV}$. The curve for model $\mathrm{E}$ almost coincides with that of model $\mathrm{D}$ in this case and is not shown in this case.

5. The spin correlation function of nd elastic scattering for $E_{n}=10 \mathrm{MeV}$. For other details see Caption of Fig. 3.

6. The nucleon-to-deuteron polarization transfer coefficient $K_{y}^{y^{\prime}}$ of nd elastic scattering for $E_{n}=10 \mathrm{MeV}$. For other details see Caption of Fig. 3.

7. The nucleon-to-deuteron polarization transfer coefficient $K_{x}^{x^{\prime}}$ of nd elastic scattering for $E_{n}=10 \mathrm{MeV}$. For other details see Caption of Fig. 3. 


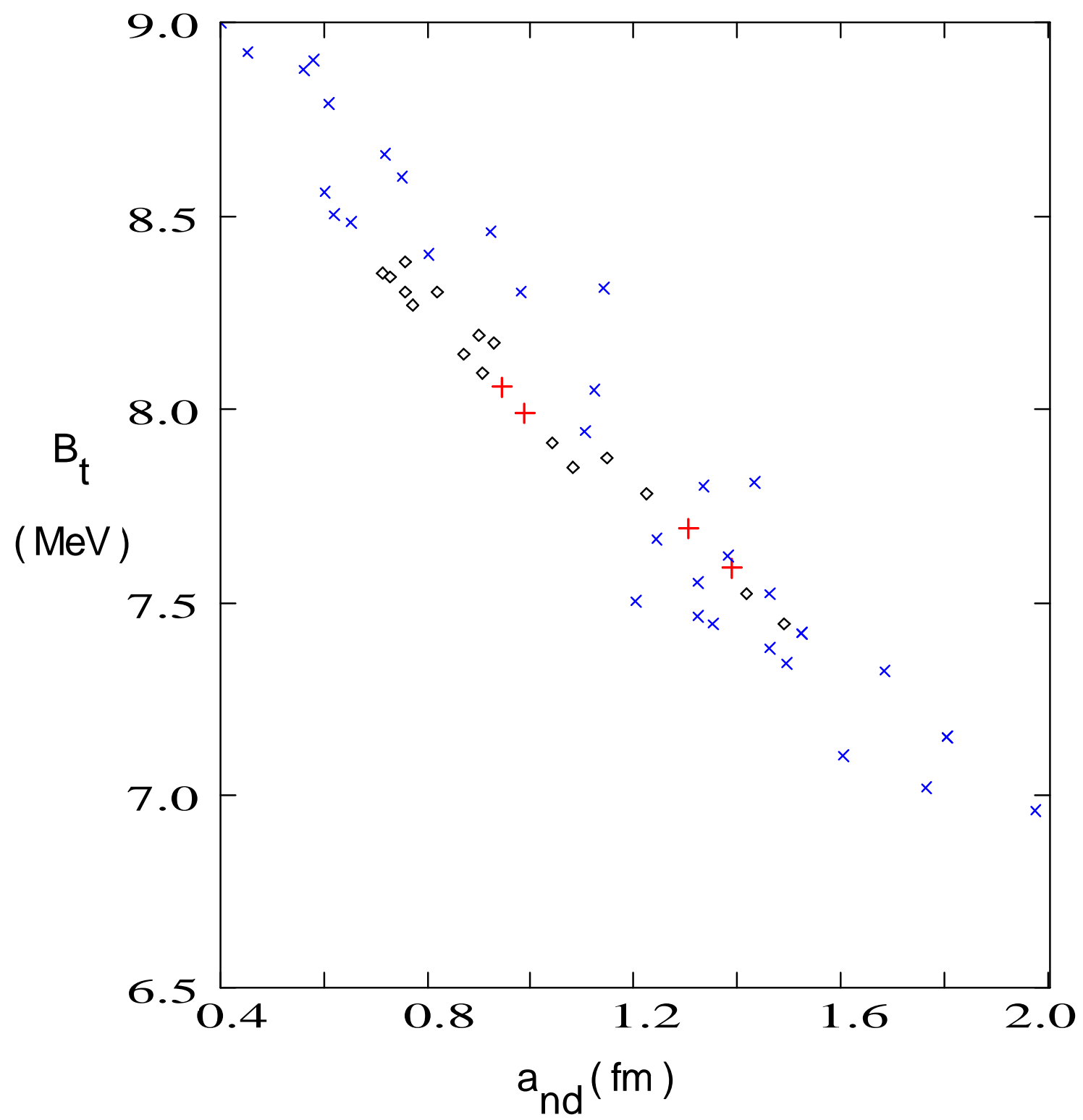

Fig. 1 
This figure "fig1-1.png" is available in "png" format from: http://arxiv.org/ps/nucl-th/9410006v2 
This figure "fig2-1.png" is available in "png" format from: http://arxiv.org/ps/nucl-th/9410006v2 


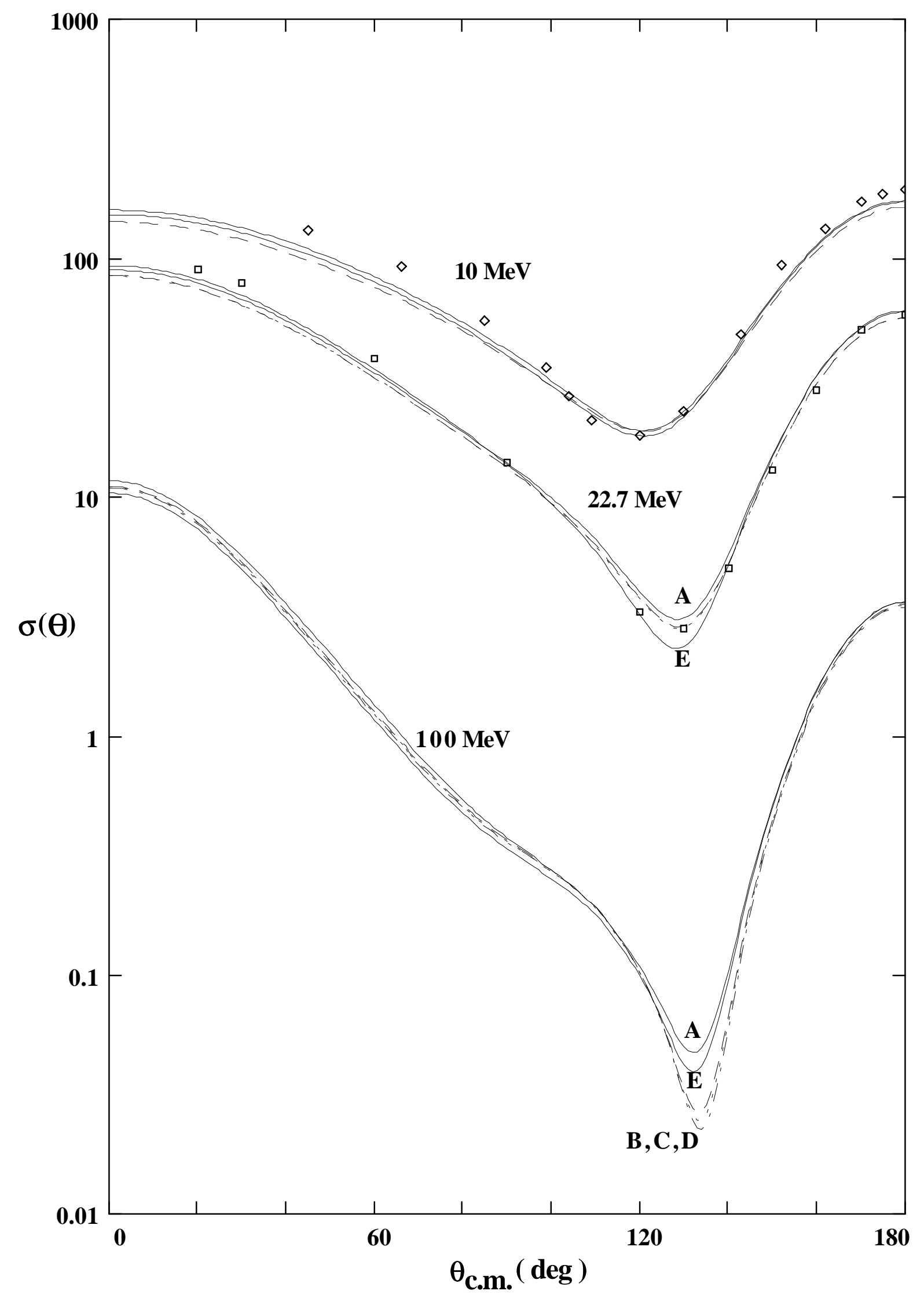

F i g . 2 

This figure "fig1-2.png" is available in "png" format from: http://arxiv.org/ps/nucl-th/9410006v2 
This figure "fig2-2.png" is available in "png" format from: http://arxiv.org/ps/nucl-th/9410006v2 


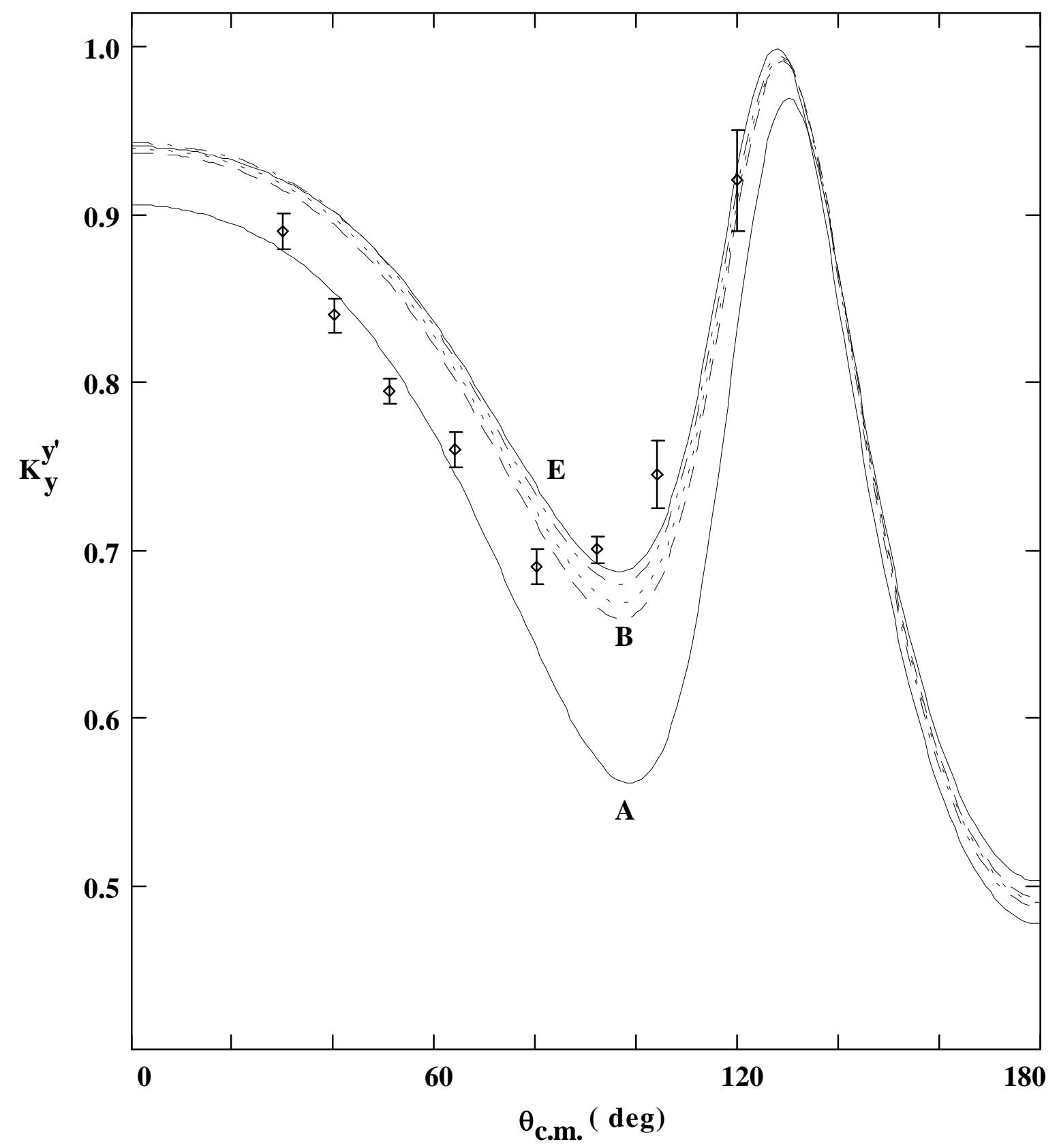

F i g . 3 

This figure "fig1-3.png" is available in "png" format from: http://arxiv.org/ps/nucl-th/9410006v2 


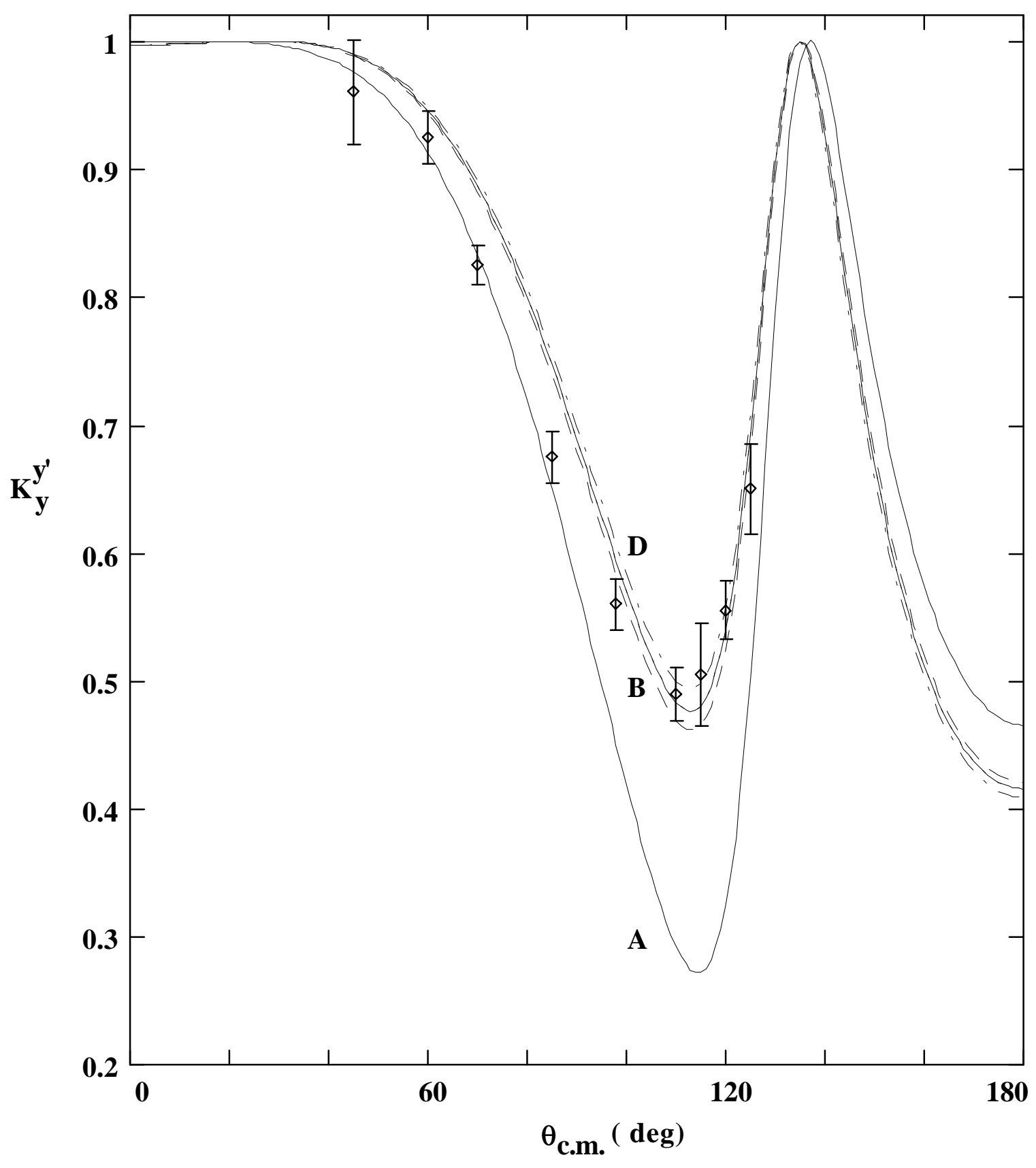

F i g . 4 

This figure "fig1-4.png" is available in "png" format from: http://arxiv.org/ps/nucl-th/9410006v2 


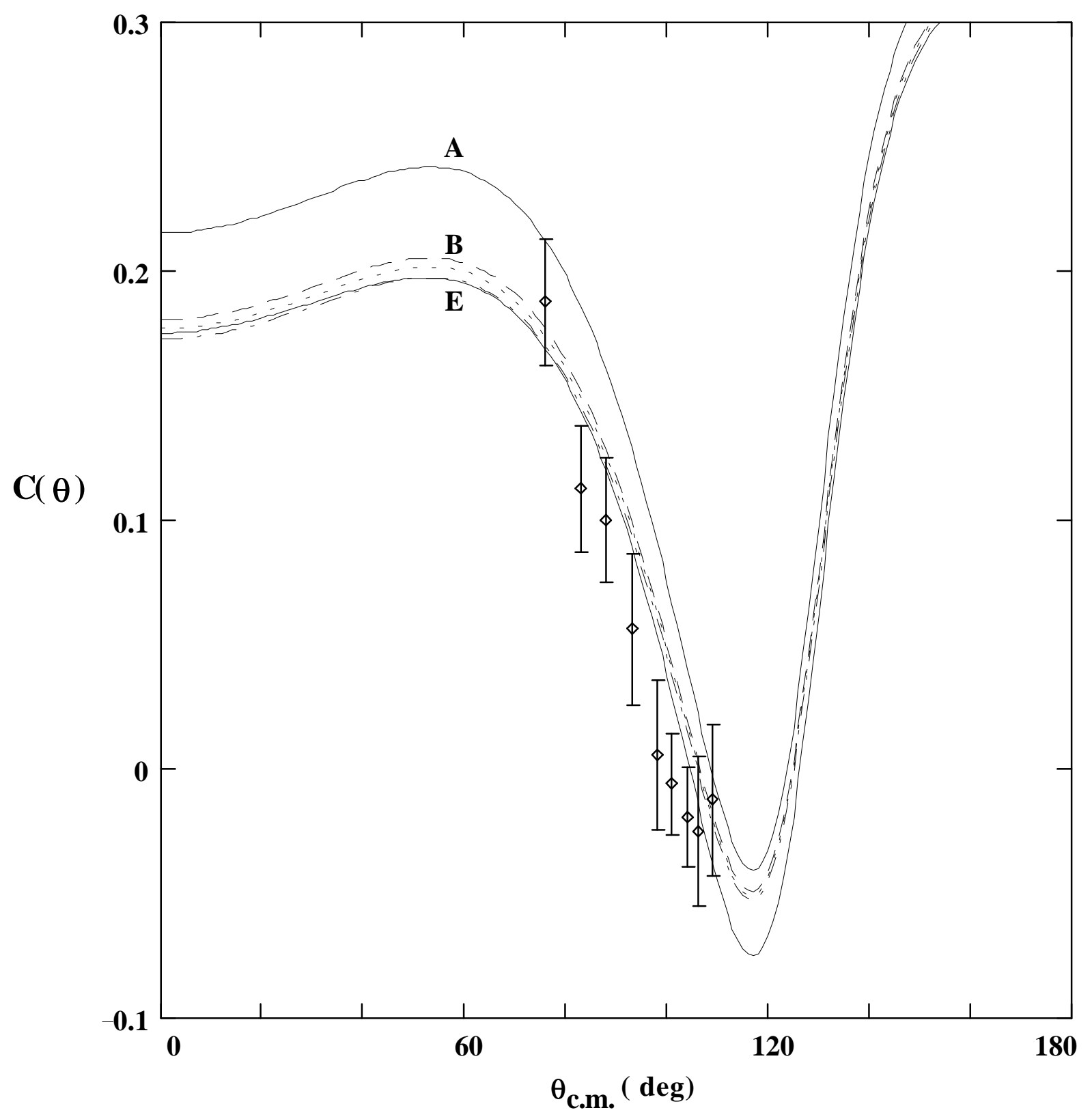

F i g . 5 

This figure "fig1-5.png" is available in "png" format from: http://arxiv.org/ps/nucl-th/9410006v2 


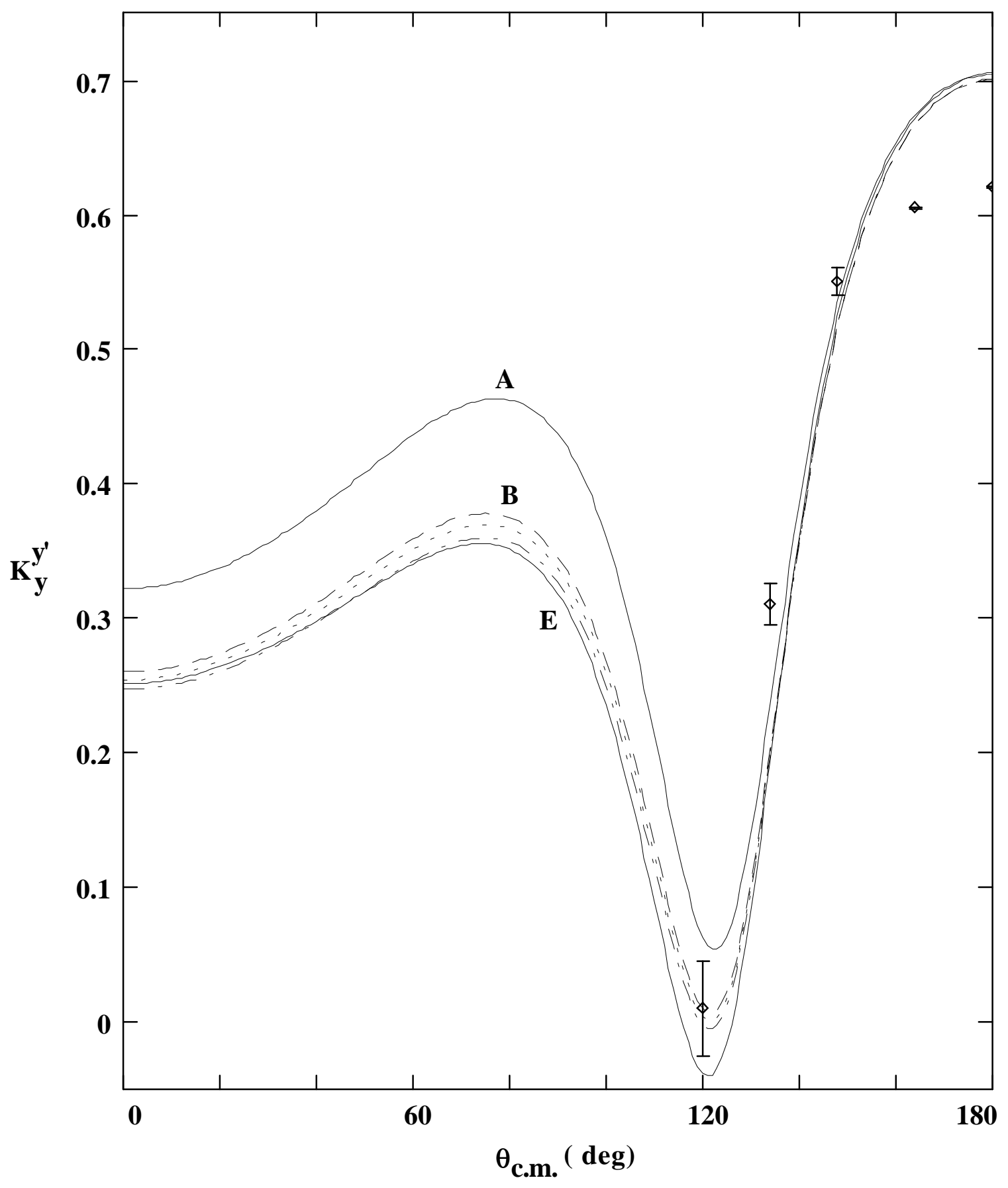

F i g . 6 



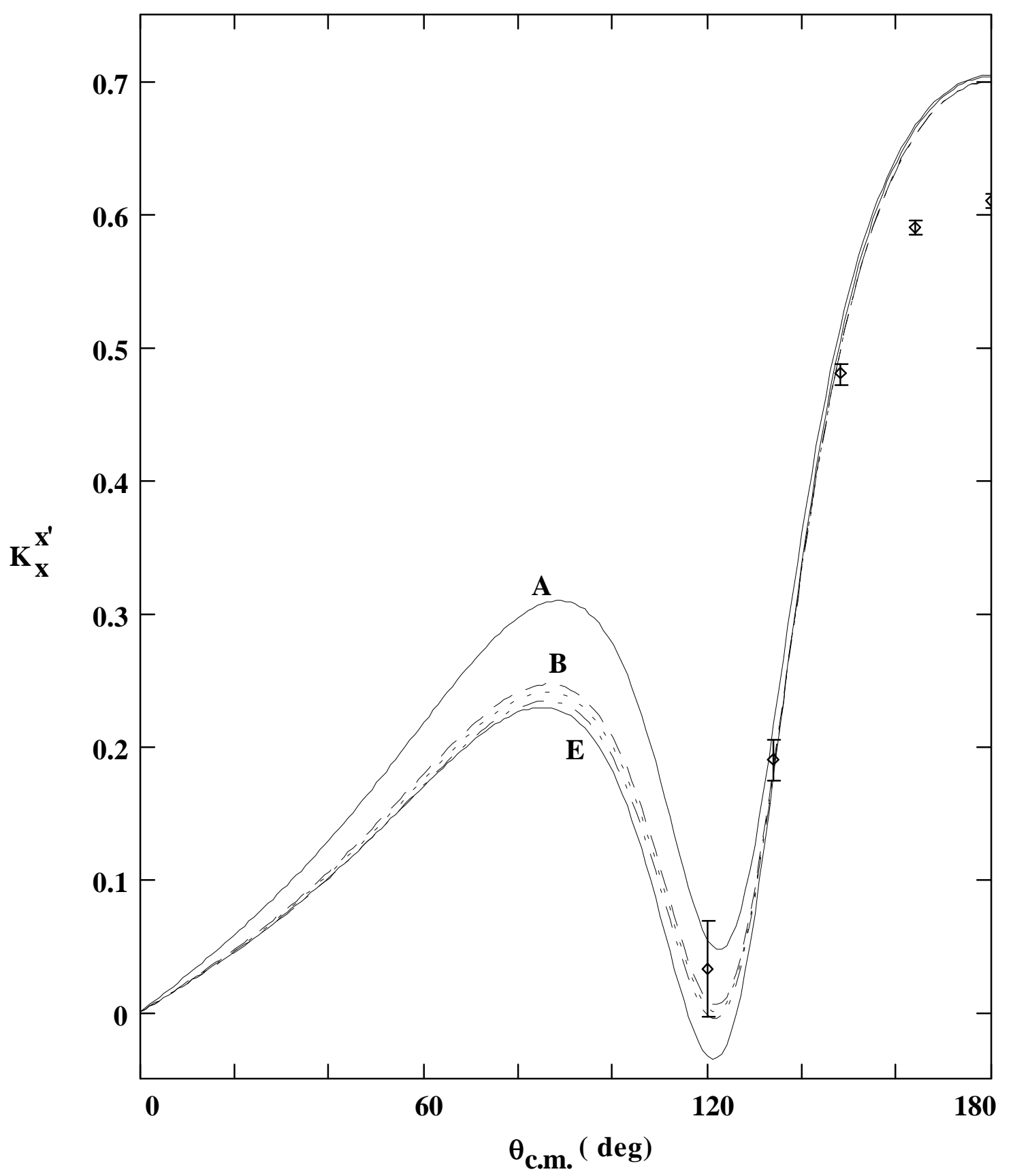

F i g . 7 
